\title{
Total synthesis of amorfrutin B via a Pd-catalyzed regioselective geranyl migration-decarboxylation-cycloaromatization cascade
}

\author{
Chao Wang, ${ }^{* a}$ Yajun Kong, ${ }^{\text {and Hongyun Qiao }}{ }^{\text {b }}$ \\ ${ }^{a}$ School of Material Science and Engineering, Southwest University of Science and Technology, \\ Mianyang, Sichuan Province 621 010, China. \\ ${ }^{b}$ Research \& Development Department, ChemPartner Inc., Zhangjiang Hi-Tech Park, Shanghai \\ 201 203, China \\ E-mail: cwang2004@gmail.com
}

DOI: $\underline{\text { http://dx.doi.org/10.3998/ark.5550190.p009.162 }}$

\begin{abstract}
A biomimetic total synthesis of amorfrutin B, a resorcylate with geranyl bibenzyl scaffold, has been completed in eight steps from 2,2,6-trimethyl-4H-1,3-dioxin-4-one. The key step utilized a highly regioselective Pd-catalyzed geranyl migration-decarboxylation-cycloaromatization cascade. The methodology should be of practical utility in the construction of geranyl bibenzyl scaffolds manifested in many natural products.
\end{abstract}

Keywords: Amorfrutin B, cascade, resorcylates, biomimetic reactions

\section{Introduction}

Molecules containing a bibenzyl unit were found to occur widely in natural products ${ }^{1-3}$ and display diversified biological activities such as antioxidant, ${ }^{4}$ antimicrobial, ${ }^{5}$ antifungal, ${ }^{6}$ antitumor, ${ }^{7}$ and cytotoxic. ${ }^{8}$ Within this class, amorfrutins were isolated in 1981 by Mitscher et $a l$. , from the ethanolic extracts of the fruits, stems, and leaves of Amorpha fruticosa, a shrub originating in North America, China and Korea. ${ }^{9,10}$ In addition to antimicrobial activities against G-positive and acid-fast microorganisms, amorfrutins were able to inhibit nuclear transcription factor-jB (NF-jB) activation and related gene expression, which is known to be related to several inflammatory diseases such as arthritis, asthma, bowel inflammation, and cancer. ${ }^{11}$ Recently this family of natural products has been discovered to exhibit antidiabetic activities. ${ }^{12}$ More specifically, amorfrutin B was identified as a partial agonist of peroxisome proliferator-activated receptor $\gamma(\operatorname{PPAR} \gamma)$ with a considerably higher affinity and $\gamma$-selectivity than that of any other amorfrutins previously reported, and similar to that of the marketed drug rosiglitazone. ${ }^{13}$ Due to its physiological profiles remarkably different from current marketed PPAR $\gamma$ drugs, and the 
predictable application prospects, this compound has found extensive research interest in many labs, for example, in pharmacodynamic and structure-activity relationship studies. ${ }^{14}$

Several syntheses of geranyl bibenzyl derivatives have been reported, the majority of strategies utilizing two-step sequences: Wittig reaction followed by alkene hydrogenation, ${ }^{15}$ benzylic metalation and subsequent alkylation ${ }^{16,17}$ from protected phenolic precursors, and more recently palladium catalyzed Sonogashira reaction of phenylacetylene and triflate precursor followed by alkyne hydrogenation. ${ }^{12}$ The geranyl moiety could be introduced by electrophilic aromatic alkylation with geraniol in the presence of $\mathrm{BF}_{3}-\mathrm{OEt}_{2}$ as catalyst or by using regioselective metalation of the 2-position with $\mathrm{n}$-BuLi or $\mathrm{KH}$ and subsequent alkylation with geranyl bromide or chloride. ${ }^{13,15,18}$ However, yields were typically low, with co-production of considerable amounts of $O$-geranylated product. ${ }^{13,15}$ Besides its geranyl bibenzyl scaffold, the molecule contains a 6-alkyl-2,4-dihydroxybenzoic acid (resorcylic acid) motif, which occurs in numerous natural products. ${ }^{19-22}$ Reported syntheses invariably require the use of protective groups and strong bases, subsequent alkylations giving low yields and poor regioselectivity.

\section{Results and Discussion}

In consideration of the fact that the molecule contains a resorcylic acid unit, we were inspired by the biomimetic synthesis of resorcylates using dioxinone chemistry, especially by Barrett's elegant discovery of the cascade reaction. ${ }^{23-27}$ Herein, we report a full description and experimental details on a flexible strategy for the total synthesis of amorfrutin B, which was adapted from resorcylates biosynthesis and should be of practical utility.

Ketoester $\mathbf{6}$ was synthesized in 3 steps from dioxinone $\mathbf{1}$ by following literature procedure. ${ }^{18}$ Regioselective acylation of ketoester $\mathbf{6}$ by Claisen condensation reaction with dihydrocinnamoyl chloride $\mathbf{8}$ in the presence of magnesium chloride and pyridine gave the corresponding diketoester-dioxinone $\mathbf{9}$ in a satisfactory yield. Subsequent reaction of diketoester-dioxinone 9 with $\mathrm{Pd}\left(\mathrm{PPh}_{3}\right)_{4}$ and cesium carbonate resulted in decarboxylative geranyl migration and formation of the resorcylate 10. Finally, phenol methylation of $\mathbf{1 0}$ and saponification of resulting 13 gave amorfrutin $B$.

The synthesis utilized a highly regioselective geranyl migration-decarboxylationcycloaromatization cascade reaction as a key step. ${ }^{23-27}$ Naturally it would be very helpful to understand the mechanism of this important transformation. According to a recent paper, ${ }^{28}$ the cascade was proposed to proceed via an interesting intermolecular pathway: $\operatorname{Pd}(0) /$ base-assisted degeranylation from a second molecule; regioselective geranylation at $\mathrm{C}-3$ center; and final decarboxylation to generate the precursor $\mathbf{1 6}$ for subsequent cycloaromatization. Geranyl-diketodioxinone $\mathbf{1 6}$ then readily undergoes intramolecular condensation to give $\mathbf{1 7}$ followed by dehydration to produce resorcylate 10. Though the published paper $^{28}$ illustrated the transformation mechanism primarily based on a prenyl-diketo-dioxinone substrate and a considerable similarity is reasonably present between prenyl- and geranyl-diketo-dioxinone 
substrates, confirmative experimental evidence concerning this mechanism still needs to be found and reported in due course.

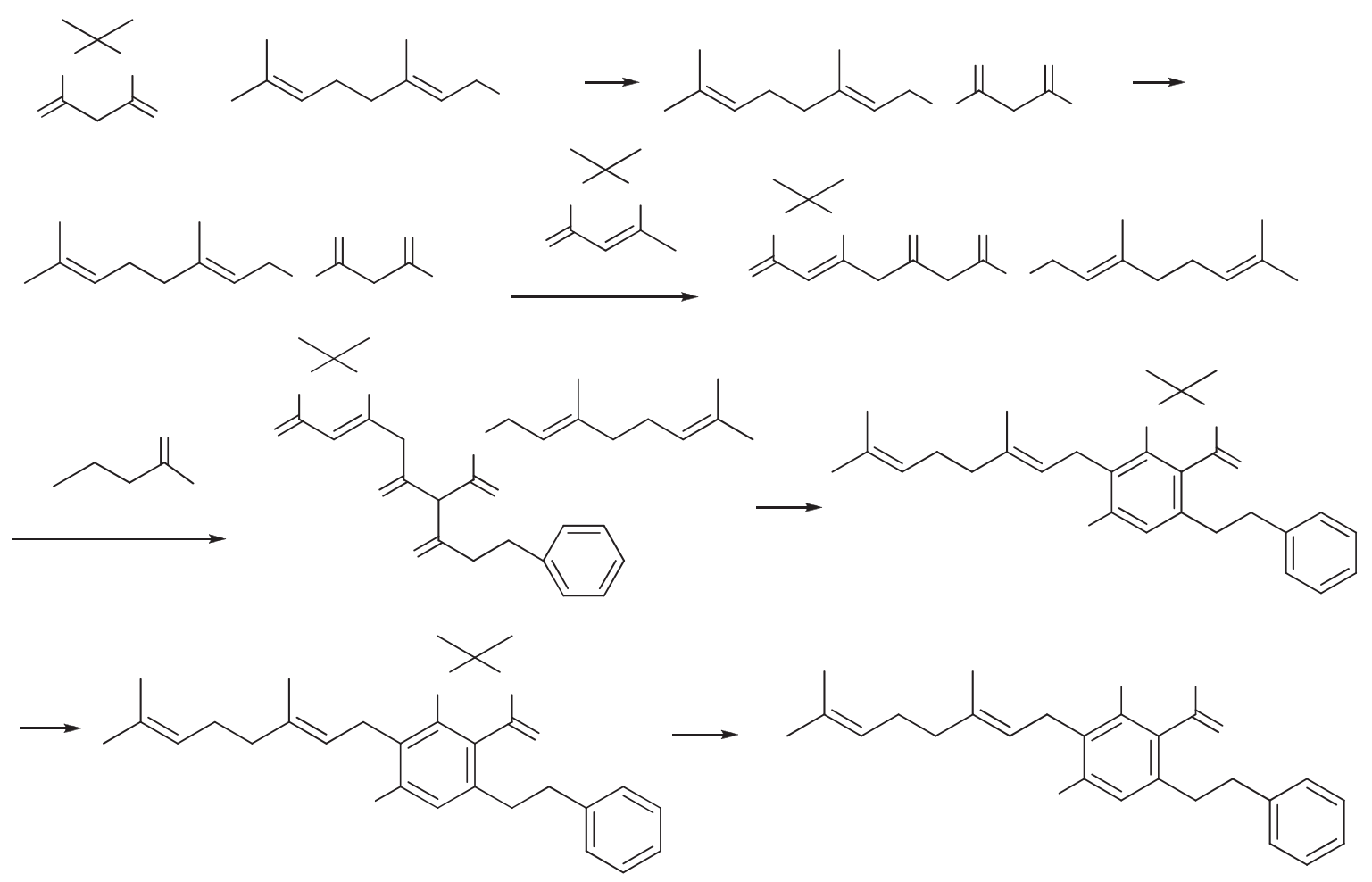

Scheme 1. Synthesis of amorfrutin B (i) PhMe, $100{ }^{\circ} \mathrm{C}, 5 \mathrm{~h}, 89 \%$; (ii) 3, isoamylene, $\mathrm{CH}_{2} \mathrm{Cl}_{2}$, r.t.; oxalyl chloride, DMF, $0{ }^{\circ} \mathrm{C}$ - r.t., 2.5 h; (iii) 5, TMSi 2 NLi, THF, $-78{ }^{\circ} \mathrm{C}, 3.5 \mathrm{~h} ; 4$, THF, -78 ${ }^{\circ} \mathrm{C}, 1.5 \mathrm{~h}, 53 \%$ over two steps; (iv) $\mathrm{MgCl}_{2}$, pyridine, $\mathrm{CH}_{2} \mathrm{Cl}_{2}, 0{ }^{\circ} \mathrm{C}, 1 \mathrm{~h} ; 8,0{ }^{\circ} \mathrm{C}, 1 \mathrm{~h}, 57 \%$; (v) $\mathrm{Pd}\left(\mathrm{PPh}_{3}\right)_{4}, \mathrm{Cs}_{2} \mathrm{CO}_{3}$, THF, $0-25^{\circ} \mathrm{C}, 18 \mathrm{~h}, 53 \%$; (vi) MeI, $\mathrm{Cs}_{2} \mathrm{CO}_{3}$, THF, $25^{\circ} \mathrm{C}, 12 \mathrm{~h}, 96 \%$; (vii) $\mathrm{KOH} 48 \%$, DMSO, $80{ }^{\circ} \mathrm{C}, 12 \mathrm{~h}, 80 \%$. 


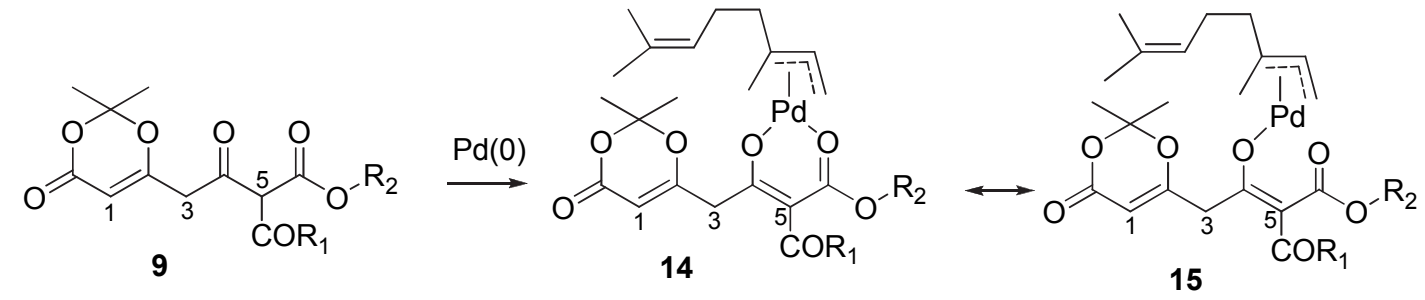<smiles>[R20]OC(=O)/C(O)=C(/C/C=C(/C)CC/C=C(/C)CC)C1=CC(=O)OC(C)(C)O1</smiles>

base $\left.\right|_{\downarrow} \mathrm{Pd}(0)$

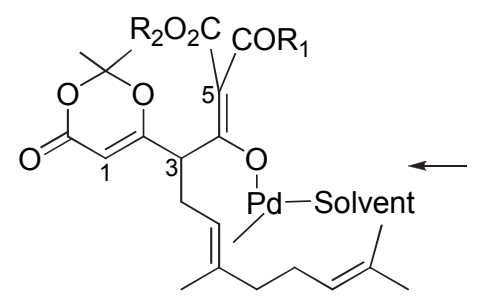

1, base

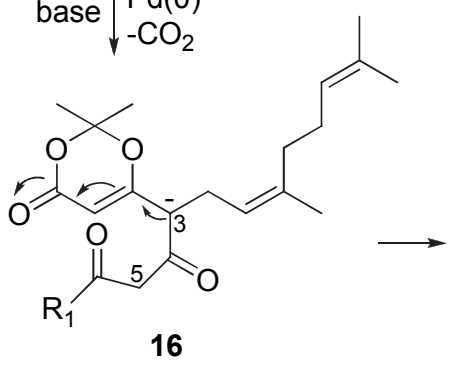<smiles></smiles>

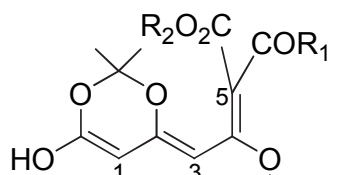<smiles>CC(=CC=CCc1cccc(C)c1)CO</smiles><smiles>[R7]C1(O)CC(=O)C(CC=C(C)CCC=C(C)C)=C2OC(C)(C)OC(=O)C21</smiles><smiles>[R7]CCCc1ccccc1</smiles>

$\mathrm{R}_{2}=$ Geranyl<smiles>C=CC(C)=CCCC(C)=CCc1c(O)cc(CCc2ccccc2)c2c1OC(C)(C)OC2=O</smiles><smiles>[R]c1cc(O)c(CC=C(C)CCC=C(C)C)c2c1C(=O)OC(C)(C)O2</smiles>

Scheme 2. Proposed mechanism for the key cascade transformation

\section{Conclusions}

The total synthesis of amorfrutin B has been accomplished in eight steps from dioxinone $\mathbf{1}$ in an overall yield of $11 \%$. We have demonstrated the efficiency of a biomimetic approach and a Pdcatalyzed geranyl migration-decarboxylation-cycloaromatization cascade to the construction of geranyl bibenzyl scaffold. In addition, the mechanism of the key cascade transformation was proposed. Further total synthesis of other resorcylates and application of the methodology to medicinal chemistry will be reported in due course. 


\section{Experimental Section}

General procedures. All reactions were carried out in oven-dried glassware under $\mathrm{N}_{2}$, using commercially supplied solvents and reagents unless otherwise stated. Column chromatography was carried out on silica gel, using flash techniques. Analytical thin layer chromatography was performed on pre-coated silica gel $\mathrm{F}_{254}$ glass plates with visualization under UV light or by staining using either acidic vanillin, anisaldehyde or ninhydrin spray reagents. ${ }^{1} \mathrm{H}$ and ${ }^{13} \mathrm{C} \mathrm{NMR}$ spectra were respectively recorded at $600 \mathrm{MHz}$ and $150 \mathrm{MHz}$ with chemical shifts $(\delta)$ quoted in parts per million (ppm) and coupling constants $(J)$ recorded in Hertz $(\mathrm{Hz})$. High-resolution ESI mass spectra were obtained on quadrupole time-of-flight instrument (Q-Star-TOF) in the electrospray-ionization mode.

(E)-3-(3,7-Dimethylocta-2,6-dienyloxy)-3-oxopropanoic acid (3). To a $50 \mathrm{~mL}$ round-bottleflask were added Meldrum's acid (1, $6.80 \mathrm{~g}, 47.0 \mathrm{mmol})$ and geraniol $(\mathbf{2}, 10.2 \mathrm{~mL}, 58.8 \mathrm{mmol})$ in toluene $(5 \mathrm{~mL})$. The mixture was refluxed with stirring for $5 \mathrm{~h}$. After cooling to room temperature, aqueous ammonia $(10 \mathrm{~mL}, 30 \%)$ was added and stirred for $10 \mathrm{~min}$. EtOAc $(30 \mathrm{~mL})$ was then added, and the two phases were separated. The aqueous layer was acidified to $\mathrm{pH} \sim 3$ with $1 \mathrm{M} \mathrm{HCl}$, and extracted with EtOAc $(3 \times 10 \mathrm{~mL})$. The combined organic layers were dried with anhydrous $\mathrm{MgSO}_{4}$, and subsequent rotary evaporation afforded 3 (10.04 g, 89\%) as a pale yellow oil, sufficiently pure for the next step. An analytically pure sample (70\%) was obtained by column chromatography as a colorless oil. $\mathrm{R}_{f} 0.27$ (EtOAc/MeOH 10:1); FT-IR (KBr) $v_{\max }$ $1745,1721,1154,980,906,827,758 \mathrm{~cm}^{-1} ;{ }^{1} \mathrm{H}$ NMR $\left(\mathrm{CDCl}_{3}\right) \delta 8.60$ (brs, $\left.1 \mathrm{H}\right), 5.35$ (t, $J=7.3$ $\mathrm{Hz}, 1 \mathrm{H}), 5.08(\mathrm{t}, J=6.1 \mathrm{~Hz}, 1 \mathrm{H}), 4.70(\mathrm{~d}, J=7.2 \mathrm{~Hz}, 2 \mathrm{H}), 3.44(\mathrm{~s}, 2 \mathrm{H}), 2.13-2.05(\mathrm{~m}, 4 \mathrm{H}), 1.71$ $(\mathrm{s}, 3 \mathrm{H}), 1.68(\mathrm{~s}, 3 \mathrm{H}), 1.60(\mathrm{~s}, 3 \mathrm{H}) ;{ }^{13} \mathrm{C}$ NMR $\left(\mathrm{CDCl}_{3}\right) \delta 171.5,166.9,143.4,131.8,122.2,117.4$, $62.8,40.8,39.5,26.2,25.6,17.6,16.5$. These spectroscopic details are consistent with literature data. $^{27}$

(E)-3,7-Dimethylocta-2,6-dienyl 3-chloro-3-oxopropanoate (4). Acid 3 (1.00 g, $4.16 \mathrm{mmol}$ ) in $\mathrm{CH}_{2} \mathrm{Cl}_{2}(5 \mathrm{~mL})$ was added with stirring to a $25 \mathrm{~mL}$ round-bottle-flask containing isoamylene (4.4 $\mathrm{mL}, 41.6 \mathrm{mmol})$ at room temperature. After cooling to $0{ }^{\circ} \mathrm{C}$, oxalyl chloride $(0.50 \mathrm{~mL}, 5.72$ mmol) and a drop of DMF were added sequentially. After stirring for $30 \mathrm{~min}$ at $0{ }^{\circ} \mathrm{C}$ and $2 \mathrm{~h}$ at room temperature, the mixture was rotary evaporated, and the residue was dried under vacuum to obtain the crude acid chloride 4 as a brown oil, to be used immediately at the next step.

(E)-3,7-Dimethylocta-2,6-dienyl 4-(2,2-dimethyl-4-oxo-4H-1,3-dioxin-6-yl)-3-oxobutanoate (6). To a solution of dioxinone $5(0.9 \mathrm{~mL}, 6.25 \mathrm{mmol})$ in THF $(2 \mathrm{~mL})$ was added fresh lithium bis(trimethylsilyl) amide $(6.7 \mathrm{~mL}, 6.7 \mathrm{mmol})$ at $-78{ }^{\circ} \mathrm{C}$ under $\mathrm{N}_{2}$. After stirring for $3.5 \mathrm{~h}$, the crude acyl chloride $4(1.08 \mathrm{~g})$ in THF $(2 \mathrm{~mL})$ was added dropwise. After $1.5 \mathrm{~h}$, saturated aqueous $\mathrm{NH}_{4} \mathrm{Cl}(10 \mathrm{~mL})$ was added to quench the reaction, and the mixture was allowed to warm to room temperature. Then $\mathrm{Et}_{2} \mathrm{O}(10 \mathrm{~mL})$ was added to dilute the solution, and the $\mathrm{pH}$ was adjusted to $\sim 2$ with $\mathrm{HCl}(1 \mathrm{M})$. The two phases were separated, and the aqueous layer was extracted with $\mathrm{Et}_{2} \mathrm{O}$ $(3 \times 10 \mathrm{~mL})$. The combined organic layers were dried with anhydrous $\mathrm{MgSO}_{4}$, rotary evaporated, and chromatographed on silica gel to obtain $\beta$-keto ester $6(0.59 \mathrm{~g}, 53 \%$ over two steps from acid 
3) as a yellow oil: $\mathrm{R}_{\mathrm{f}} 0.22$ (hexane/Et $\mathrm{E}_{2} \mathrm{O} 1: 1$ ); IR $v_{\max } 1720,1377,1271,1201,1016 \mathrm{~cm}^{-1} ;{ }^{1} \mathrm{H}$ NMR $\left(\mathrm{CDCl}_{3}\right) \delta 5.36(\mathrm{~s}, 1 \mathrm{H}), 5.35(\mathrm{mc}, 1 \mathrm{H}), 5.06(\mathrm{mc}, 1 \mathrm{H}), 4.67(\mathrm{~d}, J=7.2 \mathrm{~Hz}, 1 \mathrm{H}), 4.66(\mathrm{~d}, J=$ $7.2 \mathrm{~Hz}, 1 \mathrm{H}), 3.51$ (s, 2H), 3.50 (s, 2H), $2.10-2.05$ (m, 4H), 1.70 (s, 9H), 1.67 (s, 3H), 1.59 (s, $3 \mathrm{H}) ;{ }^{13} \mathrm{C} \mathrm{NMR}\left(\mathrm{CDCl}_{3}\right) \delta 195.7,166.4,163.6,160.5,143.6,131.9,123.5,117.3,107.3,97.1$, 62.6, 49.1, 46.9, 39.5, 32.2, 26.2, 25.7, 24.9, 17.7, 16.5; HRMS (ESI) m/z calc. $\mathrm{C}_{20} \mathrm{H}_{28} \mathrm{O}_{6}:[\mathrm{M}+$ $\mathrm{H}]^{+} 365.1962$; found 365.1978 . These spectroscopic data are consistent with literature. ${ }^{27}$

3-Phenylpropanoyl chloride $(8)$. $\mathrm{SOCl}_{2}(0.7 \mathrm{~mL}, 7.5 \mathrm{mmol})$ was added dropwise into a $25 \mathrm{~mL}$ round-bottle-flask containing 3-phenylpropanoic acid $(1.0 \mathrm{~g}, 6.8 \mathrm{mmol})$ in $\mathrm{CHCl}_{3}(10 \mathrm{~mL})$. The mixture was refluxed for $3 \mathrm{~h}$ and rotary evaporated. Then the residue was dried under vacuum to obtain the crude acid chloride $\mathbf{8}$ for immediate use at the next step.

(E)-3,7-Dimethylocta-2,6-dienyl 2-(2-(2,2-dimethyl-4-oxo-4H-1,3-dioxin-6-yl)acetyl)-3-oxo5-phenylpentanoate (9). Dioxinone $6(200 \mathrm{mg}, 0.55 \mathrm{mmol})$ in $\mathrm{CH}_{2} \mathrm{Cl}_{2}(2 \mathrm{~mL})$ was added with stirring to $\mathrm{MgCl}_{2}(52 \mathrm{mg}, 0.55 \mathrm{mmol})$ and pyridine $(0.1 \mathrm{~mL}, 1.1 \mathrm{mmol})$ in $\mathrm{CH}_{2} \mathrm{Cl}_{2}(2 \mathrm{~mL})$ at 0 ${ }^{\circ} \mathrm{C}$. After $1 \mathrm{~h}, 3$-phenylpropanoyl chloride $8(110 \mathrm{mg}, 0.68 \mathrm{mmol})$ was added dropwise. The mixture was stirred at $0{ }^{\circ} \mathrm{C}$ for $1 \mathrm{~h}$, and then brine $(10 \mathrm{~mL})$ was added to dilute the solution. The two phases were separated and the aqueous was extracted with EtOAc $(2 \times 30 \mathrm{~mL})$. The combined organic layers were dried with anhydrous $\mathrm{MgSO}_{4}$, rotary evaporated and column chromatographed to afford diketoester-dioxinone $9(155 \mathrm{mg}, 57 \%)$ as a yellow oil: $\mathrm{R}_{\mathrm{f}} 0.15$ $\left(\mathrm{Et}_{2} \mathrm{O} /\right.$ hexane 1:1); FT-IR (KBr) $v_{\max } 1726,1643,1569,1392,1357,1272,1253,1204,1175$, 1031, $900 \mathrm{~cm}^{-1} ;{ }^{1} \mathrm{H}$ NMR $\left(\mathrm{CDCl}_{3}\right) \delta 7.28-7.19(\mathrm{~m}, 5 \mathrm{H}), 5.38(\mathrm{~m}, 1 \mathrm{H}), 5.35(\mathrm{~s}, 1 \mathrm{H}), 5.28(\mathrm{~s}$, 1H), $5.05(\mathrm{mc}, 1 \mathrm{H}), 4.73(\mathrm{~d}, J=7.4 \mathrm{~Hz}, 2 \mathrm{H}), 3.69(\mathrm{~s}, 2 \mathrm{H}), 3.04-2.95(\mathrm{~m}, 4 \mathrm{H}), 2.07$ - 2.05 (m, $4 \mathrm{H}), 1.71-1.59(\mathrm{~s}, 15 \mathrm{H}) ;{ }^{13} \mathrm{C} \mathrm{NMR}\left(\mathrm{CDCl}_{3}\right) \delta 197.5,192.5,165.2,165.1,160.8,140.3,129.0$, 128.5 (2C), 128.3 (2C), 128.2, 123.5, 117.4, 108.7, 107.2, 96.5, 62.0, 61.8, 42.7, 39.5, 39.3, 32.2, 31.7, 26.2, 25.7, 24.9, 17.7, 16.5; HRMS (ESI) m/z calc. $\mathrm{C}_{29} \mathrm{H}_{36} \mathrm{O}_{7}:[\mathrm{M}+\mathrm{Na}]^{+}$519.2359; found 519.2378 .

\section{(E)-8-(3,7-Dimethylocta-2,6-dienyl)-7-hydroxy-2,2-dimethyl-5-phenethyl-4H-1,3-benzodi-} oxin-4-one (10). $\mathrm{Pd}\left(\mathrm{PPh}_{3}\right)_{4}(8.0 \mathrm{mg}, 0.007 \mathrm{mmol})$ and $\mathrm{Cs}_{2} \mathrm{CO}_{3}(75 \mathrm{mg}, 0.21 \mathrm{mmol})$ were stirred in THF $(1 \mathrm{~mL})$ for $10 \mathrm{~min}$ at $0{ }^{\circ} \mathrm{C}$. Dioxinone $9(30 \mathrm{mg}, 0.06 \mathrm{mmol})$ in THF $(1 \mathrm{~mL})$ was added dropwise into the solution. Then the mixture was allowed to stand $18 \mathrm{~h}$ at $25^{\circ} \mathrm{C}$ before brine $(10$ $\mathrm{mL}$ ) was added. The mixture was then acidified to $\mathrm{pH} \sim 2$ with $\mathrm{HCl}(1 \mathrm{M})$. The two phases were separated and the aqueous was extracted with EtOAc $(3 \times 10 \mathrm{~mL})$. The combined organic layers were dried with anhydrous $\mathrm{MgSO}_{4}$, rotary evaporated and column chromatographed to afford resorcylate 10 (14 mg, 53\%) as a white solid. $\mathrm{mp} 134-136{ }^{\circ} \mathrm{C}$ (from hexane). $\mathrm{R}_{\mathrm{f}} 0.28$ (hexane/EtOAc 5:1); FT-IR (KBr) $v_{\max } 1725,1684,1593,1515,1297,1211,903 \mathrm{~cm}^{-1}$; ${ }^{1} \mathrm{H}$ NMR $\left(\mathrm{CDCl}_{3}\right) \delta 7.31-7.23(\mathrm{~m}, 5 \mathrm{H}), 6.40(\mathrm{~s}, 1 \mathrm{H}), 5.21(\mathrm{t}, J=7.2 \mathrm{~Hz}, 1 \mathrm{H}), 5.06(\mathrm{t}, J=7.2 \mathrm{~Hz}, 1 \mathrm{H})$, $3.34(\mathrm{~d}, J=7.3 \mathrm{~Hz}, 1 \mathrm{H}), 3.33(\mathrm{~d}, J=7.3 \mathrm{~Hz}, 1 \mathrm{H}), 2.98-2.88(\mathrm{~m}, 4 \mathrm{H}), 2.10-2.05(\mathrm{~m}, 4 \mathrm{H}), 1.80$ $(\mathrm{s}, 6 \mathrm{H}), 1.69(\mathrm{~s}, 3 \mathrm{H}), 1.68(\mathrm{~s}, 3 \mathrm{H}), 1.60(\mathrm{~s}, 3 \mathrm{H}) ;{ }^{13} \mathrm{C} \mathrm{NMR}\left(\mathrm{CDCl}_{3}\right) \delta 160.2,156.2,152.4,146.3$, 141.9, 140.2, 132.1, 128.7 (2C), 128.6 (2C), 128.3, 126.4, 125.8, 122.8, 120.8, 113.2, 104.7, $39.7,37.5,36.7,30.7,26.4,25.7,22.7,21.9,17.7,16.2$; HRMS (ESI) m/z calc. $\mathrm{C}_{28} \mathrm{H}_{34} \mathrm{O}_{4}:[\mathrm{M}+$ $\mathrm{H}]^{+}$435.2535; found 435.2545. 
(E)-8-(3,7-Dimethylocta-2,6-dienyl)-7-methoxy-2,2-dimethyl-5-phenethyl-4H-1,3-benzodioxin-4-one (13). Phenol 10 (20 mg, $0.046 \mathrm{mmol})$, MeI $(12 \mu \mathrm{L}, 0.2 \mathrm{mmol})$ and $\mathrm{Cs}_{2} \mathrm{CO}_{3}(54 \mathrm{mg}$, $0.17 \mathrm{mmol})$ in THF $(2 \mathrm{ml})$ were stirred at $25{ }^{\circ} \mathrm{C}$ for $12 \mathrm{~h}$ before reaction was quenched with $\mathrm{H}_{2} \mathrm{O}$ $(1 \mathrm{~mL})$. The two phases were separated and the aqueous was extracted with EtOAc $(3 \times 5 \mathrm{~mL})$. The combined organic layers were dried with anhydrous $\mathrm{MgSO}_{4}$, rotary evaporated and column chromatographed to afford $\mathbf{1 3}(20 \mathrm{mg}, 96 \%)$ as a white solid. mp $95-97{ }^{\circ} \mathrm{C}$ (from hexane). $\mathrm{R}_{\mathrm{f}}$ $0.42\left(\right.$ EtOAc/hexane 1:7); ${ }^{1} \mathrm{H}$ NMR $\left(\mathrm{CDCl}_{3}\right) \delta 7.23$ - $7.15(\mathrm{~m}, 5 \mathrm{H}), 6.32(\mathrm{~s}, 1 \mathrm{H}), 5.12(\mathrm{t}, J=7.2$ $\mathrm{Hz}, 1 \mathrm{H}), 4.98(\mathrm{t}, J=7.2 \mathrm{~Hz}, 1 \mathrm{H}), 3.78(\mathrm{~s}, 3 \mathrm{H}), 3.27(\mathrm{~d}, J=7.3 \mathrm{~Hz}, 1 \mathrm{H}), 3.25(\mathrm{~d}, J=7.3 \mathrm{~Hz}, 1 \mathrm{H})$, $2.90-2.80(\mathrm{~m}, 4 \mathrm{H}), 2.02-1.97(\mathrm{~m}, 4 \mathrm{H}), 1.72(\mathrm{~s}, 6 \mathrm{H}), 1.60(\mathrm{~s}, 3 \mathrm{H}), 1.52(\mathrm{~s}, 6 \mathrm{H}) ;{ }^{13} \mathrm{C} \mathrm{NMR}$ $\left(\mathrm{CDCl}_{3}\right) \delta 160.3,156.3,152.4,146.5,141.9,140.3,132.1,128.8(2 \mathrm{C}), 128.7$ (2C), 128.4, 126.5, $125.9,122.9,120.8,113.3,104.7,56.4,39.8,37.4,36.9,30.9,26.5,25.7,22.9,22.1,17.8,16.3$; HRMS (ESI) $m / z$ calc. $\mathrm{C}_{29} \mathrm{H}_{36} \mathrm{O}_{4}:[\mathrm{M}+\mathrm{H}]^{+} 449.2692$; found 449.2680 .

Amorfrutin B. Aqueous $\mathrm{KOH}(48 \%, 28 \mathrm{mg} \mathrm{KOH}, 0.50 \mathrm{mmol})$ was added dropwise to lactone $13(20 \mathrm{mg}, 0.045 \mathrm{mmol})$ in DMSO $(2 \mathrm{~mL})$ and the mixture was heated at $80{ }^{\circ} \mathrm{C}$ for $12 \mathrm{~h}$. Upon cooling, the mixture was acidified to $\mathrm{pH} \sim 1$ with $\mathrm{HCl}(1 \mathrm{M})$. The two phases were separated and the aqueous was extracted with EtOAc $(2 \times 5 \mathrm{~mL})$. The combined organic layers were dried with anhydrous $\mathrm{MgSO}_{4}$, rotary evaporated and column chromatographed to afford amorfrutin $\mathrm{B}$ (14.7 mg, 80\%) as a white sticky solid: $\mathrm{R}_{\mathrm{f}} 0.38($ EtOAc/hexane $1: 1) ;{ }^{1} \mathrm{H}$ NMR $\left(\mathrm{CDCl}_{3}\right) \delta 7.21-7.13$ $(\mathrm{m}, 5 \mathrm{H}), 6.29(\mathrm{~s}, 1 \mathrm{H}), 5.16(\mathrm{t}, J=7.1 \mathrm{~Hz}, 1 \mathrm{H}), 4.98(\mathrm{t}, J=7.1 \mathrm{~Hz}, 1 \mathrm{H}), 3.69(\mathrm{~s}, 3 \mathrm{H}), 3.32(\mathrm{~d}, J=$ $7.3 \mathrm{~Hz}, 1 \mathrm{H}), 3.31(\mathrm{~d}, J=7.3 \mathrm{~Hz}, 1 \mathrm{H}), 2.81-2.77(\mathrm{~m}, 4 \mathrm{H}), 2.01-1.96(\mathrm{~m}, 4 \mathrm{H}), 1.72(\mathrm{~s}, 3 \mathrm{H}), 1.60$ $(\mathrm{s}, 3 \mathrm{H}), 1.51(\mathrm{~s}, 3 \mathrm{H}) ;{ }^{13} \mathrm{C} \mathrm{NMR}\left(\mathrm{CDCl}_{3}\right) \delta 157.7,155.5,141.8,141.3,137.9,131.8,128.5(2 \mathrm{C})$, 128.3 (3C), 125.9, 123.9, 122.1, 112.7, 108.9, 103.6, 55.7, 39.7, 38.0, 37.7, 26.5, 25.7, 22.1, 17.7, 16.1; HRMS (ESI) $m / z$ calc. $\mathrm{C}_{26} \mathrm{H}_{32} \mathrm{O}_{4}$ : $[\mathrm{M}+\mathrm{H}]^{+}$409.2379; found 409.2388. These spectroscopic data are consistent with literature. ${ }^{9}$

\section{Acknowledgements}

We thank the Chinese Ministry of Education for grant support (grant number 13zs1102), Southwest University of Science and Technology for NMR instrumentation and Jiangsu Normal University for HRMS spectrometry.

\section{References}

1. Dong, H.-L.; Wang, C.-L.; Guo, S.-X.; Yang, J.-S. Chem. Pharm. Bull. 2009, 57, 513. http://dx.doi.org/10.1248/cpb.57.513

2. Feng, J.-Q.; Zhang, R.-J.; Zhao, W.-M. Helv. Chim. Acta 2008, 91, 520. http://dx.doi.org/10.1002/hlca.200890056 
3. Zidorn, C.; Lohwasser, U.; Pschorr, S.; Salvenmoser, D.; Ongania, K.-H.; Ellmerer, E. P.; Börner, A.; Stuppner, H. Phytochemistry 2005, 66, 1691. http://dx.doi.org/10.1016/j.phytochem.2005.05.004

4. Zhang, X.; Xu, J.-K.; Wang, J.; Wang, N.-L.; Kurihara, H.; Kitanaka, S.; Yao, X.-S. J. Nat. Prod. 2007, 70, 24. http://dx.doi.org/10.1021/np060449r

5. Beck, S. H.; Phipps, R. K.; Perry, N. J. Nat. Prod. 2004, 67, 718. http://dx.doi.org/10.1021/np030455c

6. Zhang, Y.-Z.; Xu, G.-B.; Zhang, T. J. Asian Nat. Prod. Res. 2008, 10, 634. http://dx.doi.org/10.1080/10286020802133555

7. Speicher, A.; Groh, M.; Zapp, J.; Schaumloeffel, A.; Knauer, M.; Bringmann, G. Synlett 2009, 1852.

http://dx.doi.org/10.1055/s-0029-1217510

8. Scher, J. S.; Burgess, E. J.; Lorimer, S. D.; Perry, N. B. Tetrahedron 2002, 58, 7875. http://dx.doi.org/10.1016/S0040-4020(02)00899-2

9. Mitscher, L. A.; Park, Y. H.; Al-Shamma, A.; Hudson, P. B.; Haas, T. Phytochemistry 1981, 20,781 . http://dx.doi.org/10.1016/0031-9422(81)85174-6

10. Dat, N. T.; Lee, J.-H.; Lee, K.; Hong, Y.-S.; Kim, Y. H.; Lee, J. J. J. Nat. Prod. 2008, 71, 1696.

http://dx.doi.org/10.1021/np800383q

11. Baldwin, A. S., Jr. J. Clin. Invest. 2001, 107, 3. http://dx.doi.org/10.1172/JCI11891

12. Weidner, C.; de Groot, J. C.; Prasad, A.; Freiwald, A.; Quedenau, C.; Kliem, M.; Witzke, A.; Kodelja, V.; Han, C. T.; Giegold, S. Proc. Natl. Acad. Sci. U.S.A. 2012, 109, 7257. http://dx.doi.org/10.1073/pnas.1116971109

13. de Groot, J. C.; Weidner, C.; Krausze, J.; Kawamoto, K.; Schroeder, F. C.; Sauer, S.; Büssow, K. J. Med. Chem. 2013, 56, 1535. http://dx.doi.org/10.1021/jm3013272

14. Song, Y.-Y.; He, H.-G.; Li, Y.; Deng, Y. Tetrahedron Lett. 2013, 54, 2658. http://dx.doi.org/10.1016/j.tetlet.2013.03.042

15. Eicher, T.; Tiefensee, K.; Dönig, R.; Pick, R. Synthesis 1991, 98. http://dx.doi.org/10.1055/s-1991-26390

16. Zinsmeister, H. D.; Becker, H.; Eicher, T. Angew. Chem., Int. Ed. Engl. 1991, 30, 130. http://dx.doi.org/10.1002/anie.199101301

17. Schwaben, J.; Cordes, J.; Harms, K.; Koert, U. Synthesis 2011, 2929. http://dx.doi.org/10.1055/s-0030-1260166

18. Laclef, S.; Anderson, K.; White, A. J. P.; Barrett, A. G. M. Tetrahedron Lett. 2012, 53, 225. http://dx.doi.org/10.1016/j.tetlet.2011.11.019

19. Winssinger, N.; Barluenga, S. Chem. Commun. 2007, 22. 
http://dx.doi.org/10.1039/B610344H

20. Garbaccio, R. M.; Stachel, S. J.; Baeschlin, D. K.; Danishefsky, S. J. J. Am. Chem. Soc. 2001, 123, 10903.

http://dx.doi.org/10.1021/ja011364+

21. Fürstner, A.; Thiel, O. R.; Kindler, N.; Bartkowska, B. J. Org. Chem. 2000, 65, 7990. http://dx.doi.org/10.1021/jo0009999

22. Stob, M.; Baldwin, R. S.; Tuite, J.; Andrews, F. N.; Gillette, K. G. Nature 1962, 196, 1318. http://dx.doi.org/10.1038/1961318a0

23. Basset, J.-F.; Leslier, C.; Hamprecht, D.; White, A. J. P.; Barrett, A. G. M. Tetrahedron Lett. 2010, $51,783$.

http://dx.doi.org/10.1016/j.tetlet.2009.11.134

24. Miyatake-Ondozabal, H.; Barrett, A. G. M. Org. Lett. 2010, 12, 5573.

http://dx.doi.org/10.1021/o1102468k

25. Navarro, I.; Basset, J.-F.; Hebbe, S.; Major, S. M.; Werner, T.; Howsham, C.; Brackow, J.; Barrett, A. G. M. J. Am. Chem. Soc. 2008, 130, 10293.

http://dx.doi.org/10.1021/ja803445u

26. Calo, F.; Richardson, J.; Barrett, A. G. M. Org. Lett. 2009, 11, 4910.

http://dx.doi.org/10.1021/o1901979x

27. Cordes, J.; Calo, F.; Anderson, K.; Pfaffeneder, T.; Laclef, S.; White, A. J. P.; Barrett, A. G. M. J. Org. Chem. 2012, 77, 652.

http://dx.doi.org/10.1021/jo202354j

28. Anderson, K.; Laclef, S.; Barrett, A. G. M. Tetrahedron 2014, 70, 5569.

http://dx.doi.org/10.1016/j.tet.2014.06.098 